\title{
Runtime Extension of Low-Power Wireless Sensor Nodes Using Hybrid-Storage Units
}

\author{
Maria T. Penella, Student Member, IEEE, and Manel Gasulla, Member, IEEE
}

\begin{abstract}
The sensor nodes of wireless sensor networks remain inactive most of the time to achieve longer runtimes. Power is mainly provided by batteries, which are either primary or secondary. Because of its internal impedance, a significant voltage drop can appear across the battery terminals at the activation time of the node, thus preventing the extraction of all the energy from the battery. Additionally, internal losses can also be significant. Consequently, the runtime is reduced. The addition of a supercapacitor in parallel with the battery, thus forming a hybrid-storage device, has been proposed under pulsed loads to increase the power capabilities and reduce both the voltage drop and the internal losses at the battery. However, this strategy has not yet thoroughly been analyzed and tested in low-power wireless sensor nodes. This paper presents a comprehensive theoretical analysis that extends previous works found in the literature and provides design guidelines for choosing the appropriate supercapacitor. The analysis is supported by extensive experimental results. Two low-capacity $(<200 \mathrm{mAh})$ batteries were tested together with their hybrid-storage unit counterparts when using an electronic load as a pulsed current sink. The hybrid-storage units always achieved a higher runtime. One of the batteries was also tested using a sensor node. The runtime extension was $16 \%$ and $33 \%$ when connecting the hybrid-storage unit directly and through a dc-dc switching regulator to the sensor node, respectively.
\end{abstract}

Index Terms-Autonomous sensor, battery impedance, hybrid storage, runtime extension, sensor node, wireless sensor networks (WSNs).

\section{INTRODUCTION}

W IRELESS sensor networks (WSNs) have been proposed for multiple applications, which range from healthcare [1] to environmental monitoring [2]. They are composed of nodes that sense different parameters and relay the data to a central node. The sensor nodes are wireless measurement devices that are mainly composed of sensors and their signal conditioners, a microprocessor, and a radio-frequency transceiver. Power to the nodes is provided by primary batteries or by harvesting the energy from the environment and storing it in secondary

Manuscript received October 20, 2008; revised May 22, 2009. Current version published March 20, 2010. This work was supported in part by the Spanish Ministry of Education and Science under Contract TEC200766331/MIC and in part by the European Regional Development Fund. The work of M. T. Penella was supported by a grant from the Ministry of Education and Science of Spain on the FPU program (AP2005-2508). The Associate Editor coordinating the review process for this paper was Dr. Cesare Alippi.

The authors are with the Instrumentation, Sensors and Interfaces Group, Electronic Engineering Department, Universitat Politècnica de Catalunya, Barcelona 08860, Spain (e-mail: mpenella@eel.upc.edu; manel.gasulla@ upc.edu).

Color versions of one or more of the figures in this paper are available online at http://ieeexplore.ieee.org.

Digital Object Identifier 10.1109/TIM.2009.2026603 (rechargeable) batteries [3]. Whatever the option, low energy consumption is desirable in increasing the autonomy of the node. Hence, sensor nodes need to be low-power devices and to remain inactive most of the time.

Batteries, which are either primary or secondary, present an internal impedance. Whenever the sensor node wakes up, a significant amount of power and then energy can be lost at this internal impedance. Moreover, the voltage drop across the internal impedance can prevent squeezing all the available charge from the battery, thus leading to a reduction in the runtime of the sensor nodes [4]. Capacitors have complementary characteristics to batteries [5], [6], and their joint use has been proposed to increase the power capabilities of batteries, even in commercial products [7]. In [8], a 600-mAh lithium battery in parallel with a $600-\mathrm{mF}$ supercapacitor was used to extend the runtime down to a specific threshold voltage under a 2-A pulse that emulated the current profile of a GSM mobile phone. The results were only experimentally assessed, and no analytical formulation was provided. Dougal et al. [9] theoretically analyzed the power and life extension under pulsed-load conditions provided by a hybridstorage unit that was composed of a supercapacitor in parallel with a battery. However, no experimental results were provided, and runtime extension was only tackled from an energetic perspective, disregarding the effect of the voltage drop at the battery terminals. In [10], a 1.2-Ah lithium-ion battery was tested in parallel with both a 5-F and a 50-F capacitance storage. The runtime of the hybrid-storage unit with the 50-F capacitor was only $5 \%$ higher than that with the single battery.

In spite of the extensive work done with hybrid-storage units, their advantages have not thoroughly been analyzed and tested in low-power wireless sensor nodes, where pulsed-load currents on the order of tens of milliamperes are present. This paper, which, in part, started in [11], presents a comprehensive theoretical analysis that extends previous works found in the literature and provides design guidelines for choosing the appropriate supercapacitor. The analysis is supported by extensive experimental results. Two low-capacity $(<200 \mathrm{mAh})$ batteries were tested together with their hybrid-storage unit counterparts when using an electronic pulsed load and a sensor node equipped with a commercial Zigbee module as loads. The performance at low temperatures $\left(-15{ }^{\circ} \mathrm{C}\right)$ and the use of a switching regulator placed between the storage unit and the sensor node are also considered. Although this paper is focused on wireless sensor nodes, the analysis and results presented can be applicable to other low-power devices that present a pulsed current-consumption profile. 


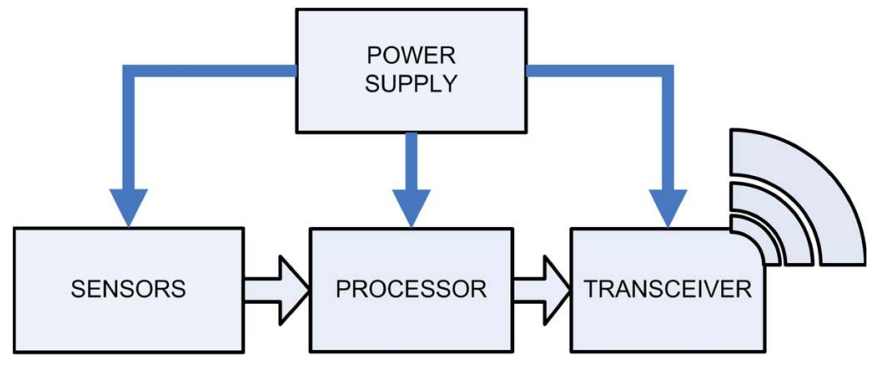

Fig. 1. Block diagram of a wireless sensor node.

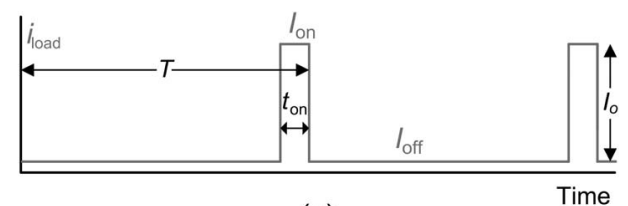

(a)

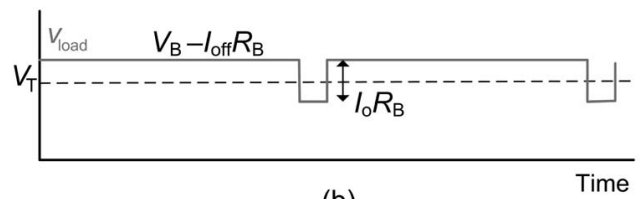

(b)

Fig. 2. (a) Current consumption of the sensor node. (b) Resulting battery voltage profile.

\section{Problem Statement}

Sensor nodes in a WSN are mainly composed of sensors, a processor, a transceiver, and a power source (Fig. 1). To save power, these nodes are inactive (sleep mode) most of the time and periodically wake up to measure and send data to a neighbor or central node. The current consumption of typical transceivers, which is usually the hungriest part, in sleep mode $\left(I_{\text {off }}\right)$ is of a few microamperes and reaches tens of milliamperes in active mode $\left(I_{\mathrm{on}}\right)$. Fig. 2(a) shows this situation, where $T, t_{\text {on }}$, and $t_{\text {off }}$ are the period, active, and sleep time intervals, respectively, of the pulsed current $i_{\text {load }}$, and $I_{\mathrm{o}}=I_{\mathrm{on}}-I_{\mathrm{off}}$. The average current is $I_{\mathrm{av}}=D I_{\mathrm{o}}+I_{\mathrm{off}}$, where $D=t_{\mathrm{on}} / T$ is the duty cycle. Although $I_{\mathrm{off}}$ is several orders of magnitude lower than $I_{\text {on }}$, its contribution will be dominant in applications where $D<I_{\text {off }} / I_{\text {on }}$.

Power to the nodes is mainly provided by batteries. Choosing a suitable battery is not always easy because of different constraints (e.g., battery voltage, temperature range, reliability, and energy density). Due to its internal impedance, a voltage drop across the battery terminals appears at the activation time (Fig. 2(b), where $V_{\mathrm{B}}$ and $R_{\mathrm{B}}$ are the open-circuit voltage (OCV) and the internal resistance of the battery, respectively). When the battery voltage falls below the minimum allowed supply voltage [ $V_{\mathrm{T}}$ in Fig. 2(b)], the sensor node stops working, thus impeding the extraction of all the available energy from the battery and then reducing the node runtime. Additionally, some power and then energy are lost at the internal impedance of the battery. One way to decrease its internal impedance is to increase the surface area for the electrochemical reaction. However, this leads to a reduced energy density and higher leakage of the battery, which decreases the runtime [4]. An

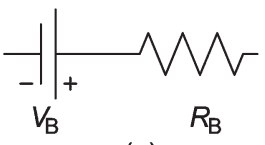

(a)

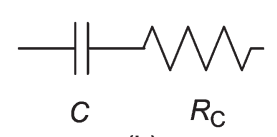

(b)
Fig. 3. Simple (a) battery and (b) supercapacitor electrical models.

alternative is to place a supercapacitor in parallel with the battery to reduce the equivalent impedance of the storage unit. This strategy is used in some commercial hybrid batteries [7], where the current capabilities of bobbin-type lithium cells are enhanced by adding a hybrid layer capacitor all within a single can. Here, generic commercial batteries and supercapacitors will be combined and tested to power low-power wireless sensor nodes.

\section{ENERGy Storage DeVices}

Two main types of batteries exist, i.e., primary and secondary or rechargeable batteries. In sensor nodes, secondary batteries are used together with energy transducers that harvest the energy from ambient sources [3]. Fig. 3(a) shows a simple electrical model for the battery, where $R_{\mathrm{B}}$ models the equivalent serial resistance. The value of $V_{\mathrm{B}}(\mathrm{OCV})$ depends on the internal stored charge (state of charge, SoC). Not included in the presented model is the self-discharge (leakage) of the battery, but its effect must be considered in unattended sensor nodes with required long runtimes. In [4], more complex models for batteries can be found.

Supercapacitors (which are also called ultracapacitors or double-layer capacitors) have a higher energy density than ordinary capacitors, i.e., they provide higher capacitance values for the same component size. They are used, for example, to provide backup power for the memory used in microcomputers and cell phones, and brief bursts of energy to numerous consumer products containing batteries, for example, in cameras [12]. Fig. 3(b) shows a simple electrical model for a supercapacitor, where $C$ is the nominal value of the capacitor, and $R_{\mathrm{C}}$ models the equivalent series resistance (ESR). The supercapacitor size increases with its nominal capacity value. In the last years, supercapacitors with very low ESRs ranging from tens to hundreds of milliohms have been commercialized. Leakage currents, which are not modeled in Fig. 3(b), have to be considered in applications with long runtimes. The same as with the batteries, leakage increases for higher temperatures.

Supercapacitors still have an energy density of one to two orders of magnitude less than that of batteries. However, its power density (internal impedance) can be much higher (lower). Their joint use (hybrid-storage unit) can provide both good energy and power density suitable for the sensor nodes of a WSN.

\section{THEORETICAL ANALYSIS}

\section{A. Circuit Model}

Fig. 4 shows the electrical model of the hybrid-storage unit and the sensor node (load), which was modeled as a pulsed current sink [Fig. 2(a)]. In the remaining part of this section, 


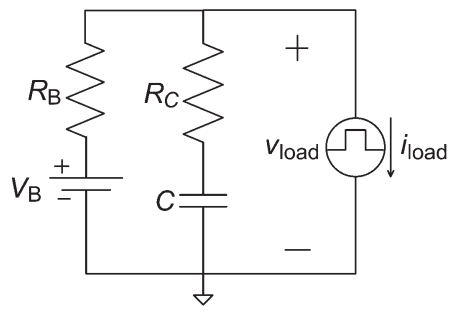

Fig. 4. Electrical model of the hybrid-storage unit and the sensor node (load).

we first calculate the voltage and power at the load and the runtime extension achieved. Then, the expressions for selecting the appropriate supercapacitor for the hybrid-storage unit are provided.

\section{B. Load Voltage and Power}

Assuming that $V_{\mathrm{B}}$ is constant and that the initial voltage at the capacitor is equal to $V_{\mathrm{B}}$, the load voltage is given by

$$
\begin{aligned}
v_{\text {load }}(t)=V_{\mathrm{B}}-\left(I_{\text {off }}+I_{\text {leak }}\right) R_{\mathrm{B}}-I_{\mathrm{o}} R_{\mathrm{B}} \\
\times \sum_{n=0}^{N}\left\{\left[1-\frac{R_{\mathrm{B}}}{R_{\mathrm{B}}+R_{\mathrm{C}}} e^{-\omega_{\beta}(t-n T)}\right] \times u(t-n T)\right. \\
-\left[1-\frac{R_{\mathrm{B}}}{R_{\mathrm{B}}+R_{\mathrm{C}}} e^{-\omega_{\beta}\left(t-n T-t_{\mathrm{on}}\right)}\right] \\
\left.\times u\left(t-n T-t_{\mathrm{on}}\right)\right\}
\end{aligned}
$$

where $I_{\text {leak }}$ is the leakage current of the storage unit, $u(t)$ is the unit-step function, $n$ is an integer that refers to the pulse number, and $\omega_{\beta}=1 /\left(\left(R_{\mathrm{B}}+R_{\mathrm{C}}\right) C\right)$. This equation is similar to that provided in [9], but now, the effect of $I_{\text {leak }}$ and $I_{\text {off }}$ has been included. The load voltage during the $N$ pulse tends to

$$
\begin{aligned}
v_{\text {load }}(N T+t)= & V_{\mathrm{B}}-\left(I_{\text {off }}+I_{\text {leak }}\right) R_{\mathrm{B}}-I_{\mathrm{o}} R_{\mathrm{B}} \\
& \times\left[1-\frac{R_{\mathrm{B}}}{R_{\mathrm{B}}+R_{\mathrm{C}}} e^{-\omega_{\beta} t}\left(\frac{1-e^{-\omega_{\beta}\left(T-t_{\mathrm{on}}\right)}}{1-e^{-\omega_{\beta} T}}\right)\right] \\
& -\left(1-\frac{R_{\mathrm{B}}}{R_{\mathrm{B}}+R_{\mathrm{C}}} e^{-\omega_{\beta} t} e^{\omega_{\beta} t_{\mathrm{on}}}\right) \\
& \times\left[u\left(t-N T-t_{\mathrm{on}}\right)-u(t-N T-T)\right]
\end{aligned}
$$

This approximation is good enough for $N T>\left(10 / \omega_{\beta}\right)$. At the end of the active time, the load voltage will be minimal and of value

$$
v_{\text {load }}\left(N T+t_{\text {on }}\right)=V_{\mathrm{B}}-\Delta v_{\text {load }}
$$

where the maximal voltage drop at the internal impedance of the battery is given by

$\Delta v_{\text {load }}=V_{\text {off_leak }}+I_{\mathrm{o}} R_{\mathrm{B}}\left[1-\frac{R_{\mathrm{B}}}{R_{\mathrm{B}}+R_{\mathrm{C}}} \frac{e^{-\omega_{\beta} t_{\mathrm{on}}}-e^{-\omega_{\beta} T}}{1-e^{-\omega_{\beta} T}}\right]$ and $V_{\text {off_leak }}=\left(I_{\text {off }}+I_{\text {leak }}\right) R_{\mathrm{B}}$. On the other hand, from (2), the average power at the load can be calculated as

$$
\begin{aligned}
& \bar{P}_{\text {load }}=\frac{1}{T} \int_{0}^{T} v_{\text {load }}(N T+t) \cdot i_{\text {load }} \cdot d t \\
&=\frac{I_{\mathrm{o}} \cdot t_{\text {on }}}{T}[ {\left[V_{\mathrm{B}}-V_{\text {off_leak }}-I_{\mathrm{o}} R_{\mathrm{B}}+I_{\mathrm{o}} \frac{R_{\mathrm{B}}^{2}}{R_{\mathrm{B}}+R_{\mathrm{C}}}\right.} \\
&\left.\times \frac{\left(1-e^{-\omega_{\beta}\left(T-t_{\mathrm{on}}\right)}\right)\left(1-e^{-\omega_{\beta} t_{\mathrm{on}}}\right)}{\omega_{\beta} t_{\text {on }}\left(1-e^{-\omega_{\beta} T}\right)}\right] \\
&+I_{\text {off }}\left(V_{\mathrm{B}}-V_{\text {off_leak }}-I_{\mathrm{o}} R_{\mathrm{B}} \frac{t_{\mathrm{on}}}{T}\right)
\end{aligned}
$$

and the average power loss will be

$$
\bar{P}_{\text {loss }}=\bar{P}_{\mathrm{B}}-\bar{P}_{\text {load }}
$$

where

$$
\bar{P}_{\mathrm{B}}=V_{\mathrm{B}}\left(I_{\mathrm{o}} \cdot D+I_{\text {leak }}+I_{\text {off }}\right)
$$

is the average power provided by the battery.

Assuming that $t_{\mathrm{on}} \ll T$ and considering $C$ and then $\omega_{\beta}$, as a design parameter, (4) and (6) can asymptotically be evaluated in three zones, i.e.,

$$
\begin{aligned}
& \text { Zone } 1: \omega_{\beta} t_{\text {on }} \gg 1, \quad \omega_{\beta} T \gg 1 \\
& \text { Zone } 2: \omega_{\beta} t_{\text {on }} \ll 1, \quad \omega_{\beta} T \gg 1 \\
& \text { Zone } 3: \omega_{\beta} t_{\text {on }} \ll 1, \quad \omega_{\beta} T \ll 1 .
\end{aligned}
$$

As $C$ increases ( $\omega_{\beta}$ decreases), we move from zones 1 to 3 .

Evaluating (4) in these three zones results in the three asymptotes plotted in Fig. 5. The respective expressions are given by

$$
\begin{aligned}
\text { Zone } 1 \rightarrow \Delta v_{\text {load }}= & V_{\text {off_leak }}+I_{\mathrm{o}} R_{\mathrm{B}} \\
= & V_{\text {off_leak }}+I_{\mathrm{o}}\left(R_{\mathrm{B}} / / R_{\mathrm{C}}\right) \\
& +I_{\mathrm{o}} \frac{R_{\mathrm{B}}^{2}}{R_{\mathrm{B}}+R_{\mathrm{C}}} \\
\text { Zone } \rightarrow \Delta v_{\text {load }}= & V_{\text {off_leak }}+I_{\mathrm{o}}\left(R_{\mathrm{B}} / / R_{\mathrm{C}}\right) \\
& +I_{\mathrm{o}} \frac{R_{\mathrm{B}}^{2}}{R_{\mathrm{B}}+R_{\mathrm{C}}} \omega_{\beta} t_{\text {on }} \\
= & V_{\text {off_leak }}+I_{\mathrm{o}}\left(R_{\mathrm{B}} / / R_{\mathrm{C}}\right) \\
& +I_{\mathrm{o}} \frac{R_{\mathrm{B}}^{2}}{R_{\mathrm{B}}+R_{\mathrm{C}}} \omega_{\beta} D T \\
\text { Zone 3 } \rightarrow \Delta v_{\text {load }}= & V_{\text {off_leak }}+I_{\mathrm{o}}\left(R_{\mathrm{B}} / / R_{\mathrm{C}}\right) \\
& +I_{\mathrm{o}} \frac{R_{\mathrm{B}}^{2}}{R_{\mathrm{B}}+R_{\mathrm{C}}} D .
\end{aligned}
$$

As an example, Fig. 6 shows the load voltage profile at zone 2, where the initial voltage drop and $\Delta v_{\text {load }}$ can be seen. As $C$ increases (we move from zones 1 to 3 ), $\Delta v_{\text {load }}$, as well as the peak current provided by the battery, decreases. In zone 1 , 


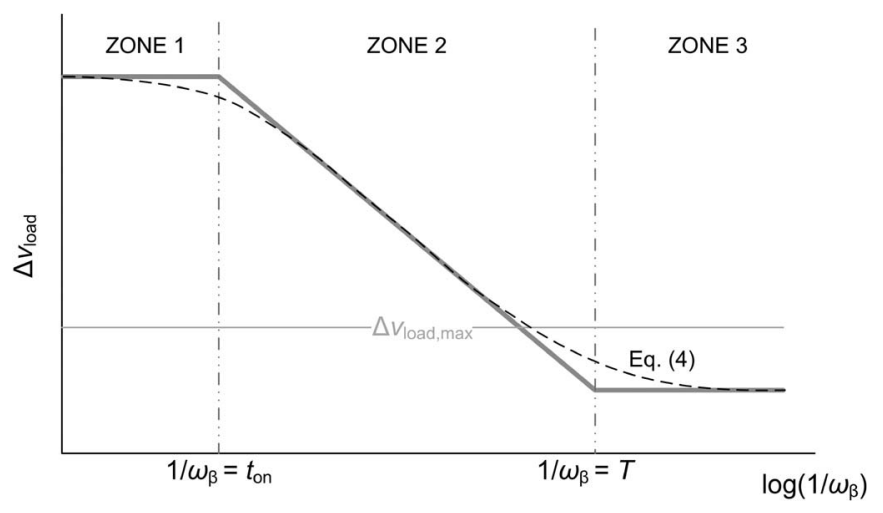

Fig. 5. $\Delta v_{\text {load }}$ versus $1 / \omega_{\beta} . \Delta v_{\text {load }}$ decreases as $C$ increases.

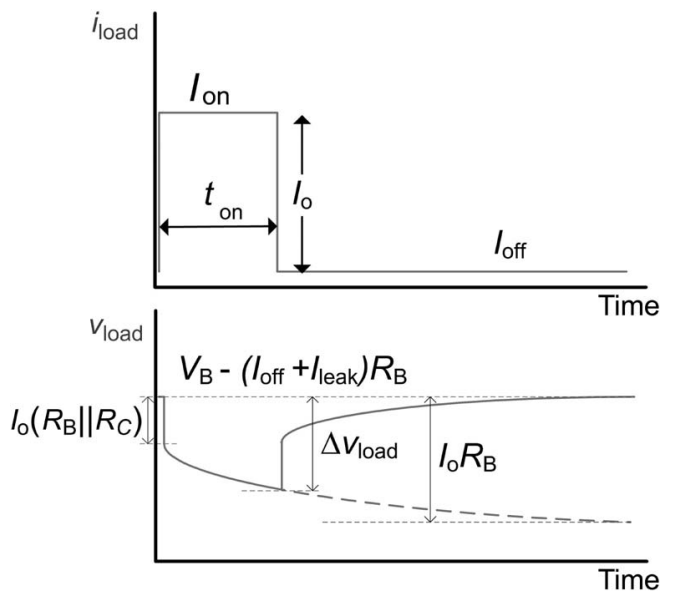

Fig. 6. Transient behavior of the load voltage under the pulsed-load current.

the hybrid-storage unit behaves as a single battery. In zone 3, the voltage drop is minimal and decreases with decreasing values of $R_{\mathrm{C}}$ and $D$.

From (5)-(7), the resulting expressions for the power loss at the three zones are given by

$$
\begin{aligned}
& \text { Zone } 1 \rightarrow \bar{P}_{\text {loss }}=I_{\mathrm{o}} D\left\lfloor V_{\text {off_leak }}+I_{\mathrm{o}} R_{\mathrm{B}}\right\rfloor+P_{\text {off_leak }} \\
& \text { Zone } 2 \rightarrow \bar{P}_{\text {loss }}=I_{\mathrm{o}} D\left[V_{\text {off_leak }}+I_{\mathrm{o}}\left(R_{\mathrm{B}} / / R_{\mathrm{C}}\right)\right. \\
& \left.\quad+I_{\mathrm{o}} \frac{R_{\mathrm{B}}^{2}}{R_{\mathrm{B}}+R_{\mathrm{C}}} \frac{t_{\text {on }}}{2} \omega_{\beta}\right]+P_{\text {off_leak }}
\end{aligned}
$$

$$
\begin{aligned}
\text { Zone } 3 \rightarrow \bar{P}_{\text {loss }}=I_{\mathrm{o}} D & {\left[V_{\text {off_leak }}+I_{\mathrm{o}}\left(R_{\mathrm{B}} / / R_{\mathrm{C}}\right)\right.} \\
& \left.+I_{\mathrm{o}} \frac{R_{\mathrm{B}}^{2}}{R_{\mathrm{B}}+R_{\mathrm{C}}} D\right]+P_{\text {off_leak }}
\end{aligned}
$$

where

$$
P_{\text {off_leak }}=I_{\text {off }}\left\lfloor V_{\text {off_leak }}+I_{\mathrm{o}} R_{\mathrm{B}} D\right\rfloor+I_{\text {leak }} V_{\mathrm{B}} .
$$

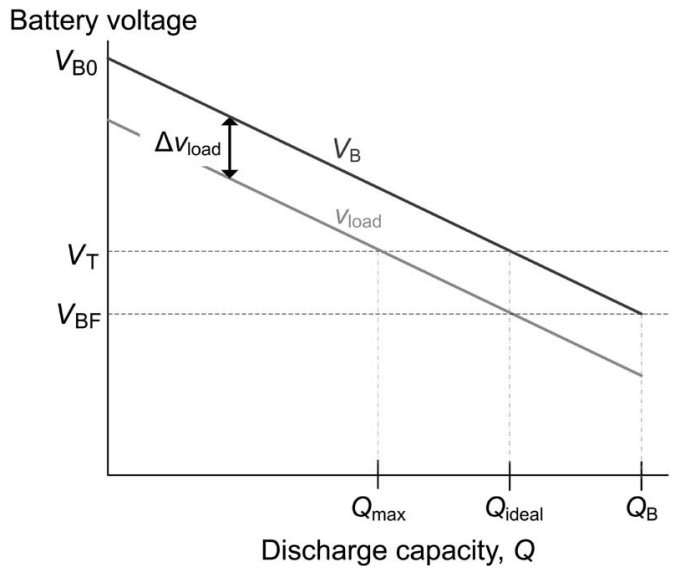

Fig. 7. Linear approximation of $V_{\mathrm{B}}$ and $v_{\text {load }}$ versus discharge capacity.

This results to a graph analogous to that of Fig. 5; thus, $\bar{P}_{\text {loss }}$ will be minimal in zone 3 .

\section{Runtime Extension}

In Section IV-B, $V_{\mathrm{B}}$ was assumed to be constant, but it actually decreases with the amount of extracted charge $Q$. Manufacturers usually provide graphs of the battery voltage versus the amount of extracted charge. Then, the runtime until the battery voltage falls below a specific threshold voltage can be estimated. The runtime increases as either the discharge current or the threshold voltage decreases. As a first-order approximation and within a determined range, $V_{\mathrm{B}}$ can be assumed to linearly decrease with $Q$; thus

$$
V_{\mathrm{B}}(t)=V_{\mathrm{B} 0}-k Q(t)
$$

where $V_{\mathrm{B} 0}$ corresponds to the battery $\mathrm{OCV}$ at a full $\mathrm{SoC}$ of the battery, and $k$ (in volts per ampere-second) is a constant that can be inferred from the discharge graphs. Fig. 7 represents (3) and (11) versus $Q$. As can be seen, the shift between both graphs increases as $\Delta v_{\text {load }}$ increases. $Q_{\max }$ is the maximum charge that can be extracted down to a threshold voltage $V_{\mathrm{T}}, Q_{\text {ideal }}$ is the charge that would be extracted with $\Delta v_{\text {load }}=0$, and $Q_{\mathrm{B}}$ corresponds to the total available charge at the battery. $V_{\mathrm{BF}}$ corresponds to the battery OCV when the $\mathrm{SoC}$ is null. When $V_{\mathrm{T}}=V_{\mathrm{BF}}$, then $Q_{\text {ideal }}=Q_{\mathrm{B}}$. The relative extracted charge is given by

$$
\frac{Q_{\text {max }}}{Q_{\text {ideal }}}=1-\frac{\Delta v_{\text {load }}}{\left(V_{\mathrm{B} 0}-V_{\mathrm{T}}\right)}
$$

where $\Delta v_{\text {load }}$ can be calculated from (4), and it is assumed that $V_{\mathrm{B} 0}>V_{\mathrm{T}}>V_{\mathrm{BF}}$. The runtime $t_{\mathrm{r}}$ can be estimated as

$$
t_{\mathrm{r}}=\frac{Q_{\max }}{D I_{\mathrm{o}}+I_{\mathrm{off}}+I_{\mathrm{leak}}} .
$$

Thus, $Q_{\max }$ and the runtime increase if $\Delta v_{\text {load }}$ is reduced. The runtime also increases with a decreasing $D$. However, for small values of $D$, the contribution of $I_{\text {off }}$ and $I_{\text {leak }}$ can 
be dominant, and a further increase in $t_{\mathrm{r}}$ is not possible. A minimum $\Delta v_{\text {load }}$ (Fig. 5 zone 3 ) is achieved whenever

$$
C \gg \frac{T}{\left(R_{\mathrm{C}}+R_{\mathrm{B}}\right)} .
$$

From (8c), a capacitor with a low $R_{\mathrm{C}}$ further reduces $\Delta v_{\text {load }}$. A reduction in $D$, by either reducing $t_{\text {on }}$ or increasing $T$, also reduces $\Delta v_{\text {load }}$. However, from (14), an increase in $T$ leads to an increase in $C$ and, then, in its size, and, for the same type and manufacturer, an increase in its cost. Working at zone 2 (Fig. 5) can lead to a smaller value and size of $C$. This will be feasible whenever the achieved $\Delta v_{\text {load }}$ permits obtaining the desired charge $Q_{\max }$ from the battery. From (12), the maximum permitted voltage drop (Fig. 5, $\Delta v_{\text {load,max }}$ ) can be deduced for a desired $Q_{\max }$. Then, the optimal capacitor (minimum value) can be calculated from ( $8 b)$ as

$$
C>\frac{I_{\mathrm{o}} t_{\text {on }}\left(\frac{R_{\mathrm{B}}}{R_{\mathrm{B}}+R_{\mathrm{C}}}\right)^{2}}{\Delta v_{\text {load,max }}-\left(I_{\text {off }}+I_{\text {leak }}\right) R_{\mathrm{B}}-I_{\mathrm{o}}\left(R_{\mathrm{C}} / / R_{\mathrm{B}}\right)}
$$

which, whenever $R_{\mathrm{C}} \ll R_{\mathrm{B}}$, can be approximated to

$$
C>\frac{I_{\mathrm{o}} t_{\text {on }}}{\Delta v_{\text {load,max }}-\left(I_{\text {off }}+I_{\text {leak }}\right) R_{\mathrm{B}}-I_{\mathrm{o}} R_{\mathrm{C}}} .
$$

As can be seen, the required value for $C$ increases with an increase in $I_{\mathrm{o}}, t_{\mathrm{on}}$, and $R_{\mathrm{C}}$, and a decrease in $\Delta v_{\text {load,max }}$.

Whenever the sensor node requires a constant voltage supply $V_{\mathrm{CC}}$ or the battery voltage does not match the supply voltage range of the sensor node, a voltage regulator must be inserted between the energy storage unit and $V_{\mathrm{CC}}$. Thus, $\bar{P}_{\text {load }}$ will be constant. From (9), $\bar{P}_{\text {loss }}$ is smaller when using a hybrid-storage unit. Then, from (6), $\bar{P}_{\mathrm{B}}$ will be reduced, and then, the runtime will further be extended.

In conclusion, the addition of a proper capacitor in parallel with high-impedance batteries increases their current and power capabilities and extends the runtime of the wireless sensor node by decreasing both its internal voltage drop, which implies that more energy can be extracted from the battery, and its power loss, which increases the efficiency of the extracted energy transferred to the load.

\section{Materials And Methods}

Accelerated (high $D$ ) runtime tests were carried out with both single batteries and hybrid-storage units. The source GS610 (Yokogawa) was used as a sink pulsed load (Fig. 2) with the following parameters: $I_{\mathrm{on}}=30 \mathrm{~mA}, I_{\mathrm{off}}=0 \mathrm{~mA}$, $t_{\mathrm{on}}=100 \mathrm{~ms}$, and $T=1 \mathrm{~s}$. Then, $I_{\mathrm{av}}=3 \mathrm{~mA}$, and $D=0.1$. In this case, $I_{\text {off }}$ and $I_{\text {leak }}$ were much lower than the mean current consumption and had a negligible effect on the runtime. The source GS610 was programmed to record the voltage and to switch off its output when the measured voltage fell below a preset voltage. A digital multimeter (Agilent 34401) was used to measure the voltage drop at the storage unit. The multimeter was configured with 4.5 digits and a high input impedance $(>10 \mathrm{G} \Omega$ ) and was triggered every $30 \mathrm{~s}$, acquiring 1000 samples during 1.6 s. Fig. 8 shows the measurement setup.

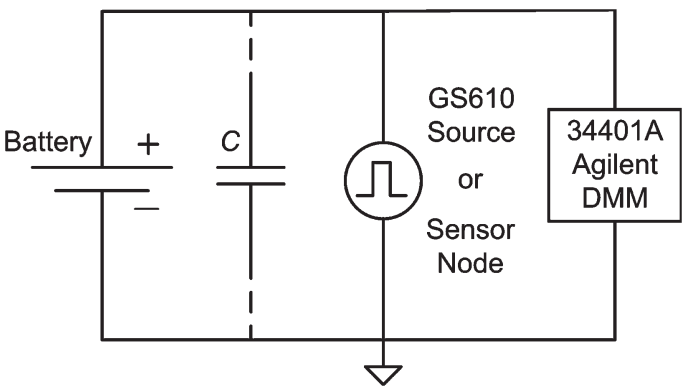

Fig. 8. Measurement setup with the storage unit and the load. Either a GS610 source or a sensor node was used as load. A multimeter was used to record the load voltage.

Table I shows the main characteristics of three selected batteries. One of them is a primary battery, and the other two are secondary batteries. $R_{\mathrm{B}}$ was measured in the following way: A 100-ms current pulse was drained from the battery with the GS610 source, and the voltage drop between the battery terminals at the end of the pulse was measured with an oscilloscope (Agilent DSO6032A). The amplitude of the current pulse was tailored to each battery. $R_{\mathrm{B}}$ was then estimated by dividing the voltage drop by the current amplitude. $\Delta v_{\text {load,max }}$ was estimated from discharge curves to extract $95 \%$ of the total capacity of the battery $Q_{\mathrm{B}}$ when the voltage fell below the end value of the voltage range, i.e., $1.4 \mathrm{~V}$ for the primary battery and $2 \mathrm{~V}$ for the secondary batteries. For the rechargeable batteries, we used the discharge curves from the manufacturer with the minimum discharge current. No discharge curve was available for the primary battery; thus, an experimental discharge curve was generated from a fresh battery.

Table II shows the main characteristics of several supercapacitors. $R_{\mathrm{C}}$ was measured with an impedance analyzer (HP 4294) at a frequency of $1000 \mathrm{~Hz}$. As can be seen, the measured values approach those specified by manufacturers. The measured values of $R_{\mathrm{C}}$ were one to two orders of magnitude lower than the measured values of $R_{\mathrm{B}}$ (Table I).

The process of estimating the appropriate value of $C$ for the hybrid-storage unit was a little bit cumbersome. First, (8a) was compared with $\Delta v_{\text {load,max }}$ for each battery (Table I). The experimental results of $R_{\mathrm{B}}$ were used to calculate (8a). Whenever the result of (8a) is lower than $\Delta v_{\text {load,max }}$, the addition of a capacitor in parallel with the battery is unnecessary, and the goal of extracting $95 \%$ of $Q_{\mathrm{B}}$ can be achieved with the single battery. This was the case of the NiMH secondary battery, which presented a low internal impedance. Second, for each of the remaining batteries, (8c) is calculated for each capacitor in Table II and compared with $\Delta v_{\text {load,max }}$. The experimental results of $R_{\mathrm{B}}$ and $R_{\mathrm{C}}$ were used to calculate (8c). When the battery voltage was over the supercapacitor maximum operating voltage, two supercapacitors connected in series were considered, and $R_{\mathrm{C}}$ was multiplied by 2 . Whenever (8c) is higher than $\Delta v_{\text {load,max }}$, the considered capacitor cannot be used. Finally, the minimum value capacitor that accomplished (15) was selected, leading to a minimum-size solution. However, other selection criteria such as the cost could be considered. When experimental values of $R_{\mathrm{B}}$ and $R_{\mathrm{C}}$ are not available, the values provided by the manufacturers can be used. 
TABLE I

Selected Batteries AND Their Main Characteristics

\begin{tabular}{llllllll}
\hline \hline Battery & Type & $\begin{array}{l}\text { Capacity } \\
(\mathrm{mAh})\end{array}$ & $\begin{array}{l}\text { Voltage } \\
\text { range }(\mathrm{V})\end{array}$ & $\begin{array}{l}\text { Nominal } \\
\text { voltage }(\mathrm{V})\end{array}$ & $\begin{array}{l}R_{\mathrm{B}}(\Omega) \\
\text { Manufacturer }\end{array}$ & $\begin{array}{l}R_{\mathrm{B}}(\Omega) \\
\text { Measured }\end{array}$ & $\begin{array}{l}\Delta v_{\text {load,max }} \\
(\mathrm{mV})\end{array}$ \\
\hline V357 (Varta) & Primary silver-oxide & 165 & $1.6-1.4$ & 1.55 & $6^{\mathrm{a}}$ & 4.79 & 20 \\
ML 2016 (Maxell) & Secondary lithium & 25 & $3.2-2$ & 3 & Not Specified & 14 & 80 \\
GP17AAAH2X ${ }^{\mathrm{b}}(\mathrm{GP})$ & Secondary NiMH & 170 & $2.9-2$ & 2.4 & $0.24^{\mathrm{c}}$ & 0.93 & 150 \\
\hline \hline
\end{tabular}

${ }^{\mathrm{a}}$ Not provided by the manufacturer; extracted from [3]. Value measured at $1000 \mathrm{~Hz}$ and $100 \% \mathrm{SoC}$.

${ }^{\mathrm{b}}$ Battery pack consisting of two series connected GP17AAAH cells.

${ }^{\mathrm{c}}$ This value is obtained multiplying by two the typical value provided by the manufacturer for the internal resistance at $1000 \mathrm{~Hz}$ of a GP17AAAH battery

TABLE II

SElected Supercapacitors AND THEIR Main CHARACTERISTICS

\begin{tabular}{lllll}
\hline \hline \multicolumn{1}{c}{ Supercapacitor } & $\begin{array}{c}\text { Capacity } \\
(\mathrm{F})\end{array}$ & $\begin{array}{c}\text { Maximum } \\
\text { operating } \\
\text { voltage (V) }\end{array}$ & $\begin{array}{c}R_{\mathrm{C}}(\Omega) \\
\text { Manufacturer } \\
(@ 1000 \mathrm{~Hz})\end{array}$ & $\begin{array}{c}R_{\mathrm{C}}(\Omega) \\
\text { Measured }\end{array}$ \\
\hline BZ series (AVX) & 0.1 & 5.5 & $0.096^{\mathrm{d}}$ & 0.095 \\
BZ series (AVX) & 1 & 5.5 & $0.050^{\mathrm{d}}$ & 0.032 \\
HW series (Panasonic) & 1 & 2.3 & $<1^{\mathrm{d}}$ & 0.215 \\
PC4 (Maxwell) & 4 & 2.5 & $0.290^{\mathrm{e}}$ & 0.120 \\
PC10 (Maxwell) & 10 & 2.5 & $0.130^{\mathrm{e}}$ & 0.105 \\
\hline \hline
\end{tabular}

${ }^{\mathrm{d}}$ Maximum value

e Typical value

TABLE III

HYBRID-STORAGE UNITS

\begin{tabular}{llll}
\hline \hline Battery & Charge Method & Capacitance (F) & $\begin{array}{c}\text { Added } \\
\text { capacity (\%) }\end{array}$ \\
\hline V357 & -- & 1 (BZ series) & 0.03 \\
ML 2016 & CCCV $^{\mathrm{f}}$ & 0.1 (BZ series) & 0.13 \\
\hline \hline
\end{tabular}

Table III shows the two resulting hybrid-storage units. The secondary battery was recharged with the charge method recommended by the manufacturer. The relative added charge introduced by the corresponding supercapacitor was computed as

$$
Q_{\mathrm{ad}}=\frac{C\left(V_{\mathrm{B}, \max }-V_{\mathrm{B}, \min }\right)}{Q_{\mathrm{B}}}
$$

where $V_{\mathrm{B}, \max }$ and $V_{\mathrm{B}, \min }$ correspond to the maximum and minimum values of the battery voltage range in Table $\mathrm{I}$, respectively, and $Q_{\mathrm{B}}$ is the (charge) capacity of the battery. In both cases, $Q_{\mathrm{ad}}$ was well below the nominal capacity of the corresponding battery; thus, it did not significantly contribute to the runtime extension. The leakage of the 0.1-F supercapacitor was measured. The supercapacitor was charged at a constant voltage $(3.3 \mathrm{~V})$, and then, after several hours of floating at this voltage, the leakage current (i.e., the current delivered by the voltage source) was measured with a KE6514 (Keithley) electrometer and was found to be $2.8 \mu \mathrm{A}$. This value is comparable to the sleep currents of commercial transceivers. Because the active currents of transceivers are about $30 \mathrm{~mA}$, only for $D<10^{-4}$ will the leakage currents be dominant. The leakage was even smaller at lower voltages: $1.4 \mu \mathrm{A}$ at $2.5 \mathrm{~V}$ and $0.6 \mu \mathrm{A}$ at $2 \mathrm{~V}$. The leakage current was also measured at different temperatures by placing the supercapacitor inside an

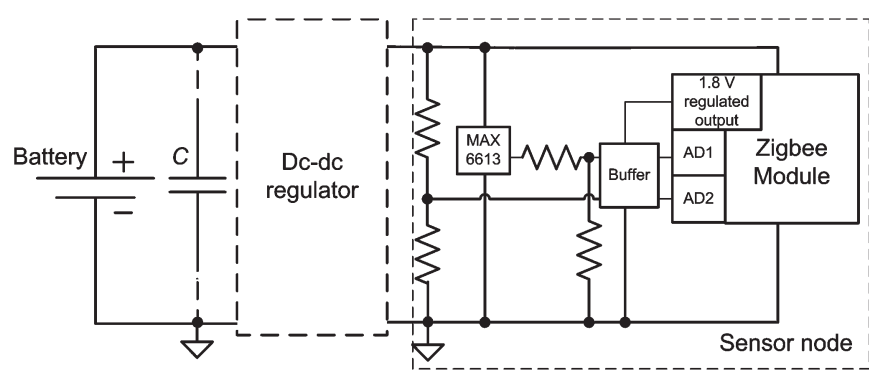

Fig. 9. Storage unit and sensor node.

FCH2730 climatic chamber (CCI). The results were $0.2 \mu \mathrm{A}$ at $-20{ }^{\circ} \mathrm{C}$ and $11.8 \mu \mathrm{A}$ at $60{ }^{\circ} \mathrm{C}$. We must consider that the leakage of primary batteries can also be at the microampere level and that it also worsens at high temperatures. Secondary batteries have even higher leakage.

Additional runtime tests were carried out using a sensor node as the load and an ML2016 lithium battery (Fig. 9). The hybridstorage unit was the same as that in the previous experiments with this battery (Table III). The sensor node was composed of a temperature sensor (MAX6613) and a Zigbee module (ETRX-2 [13], Telegesis). The Zigbee module includes a microcontroller and a transceiver. The recommended voltage supply for the module ranges from 3.6 to $2.1 \mathrm{~V}$. Tests were performed at room temperature (RT, $24{ }^{\circ} \mathrm{C}-26{ }^{\circ} \mathrm{C}$ ) and at $-15{ }^{\circ} \mathrm{C}$ with the climate chamber. At RT, tests were performed with and without a buck converter (MAX1920) placed between the storage unit and the sensor node. The output of the temperature sensor was matched to the internal 16-bit analog-to-digital converter of the module by using a suitable voltage divider. The voltage supply was also monitored by means of a high-resistance voltage divider. Buffers were used to avoid loading effects. As manufacturers recommend, $100-\mathrm{nF}$ decoupling capacitors were used for the temperature sensor and the buffer. These capacitors can be avoided, provided that the supercapacitor, when using the hybrid-storage unit, is physically close to the transceiver. The transceiver was programmed to wake up and send a data packet every $1.5 \mathrm{~s}$. Data packets were received by another transceiver node powered from the grid and connected via a serial link (EIA-232) to a computer to process the data. The distance between nodes was fixed to $35 \mathrm{~cm}$.

\section{EXPERIMENTAL RESULTS}

Fig. 10 shows the discharge profile with the silver oxide battery and the corresponding hybrid-storage unit. The source 


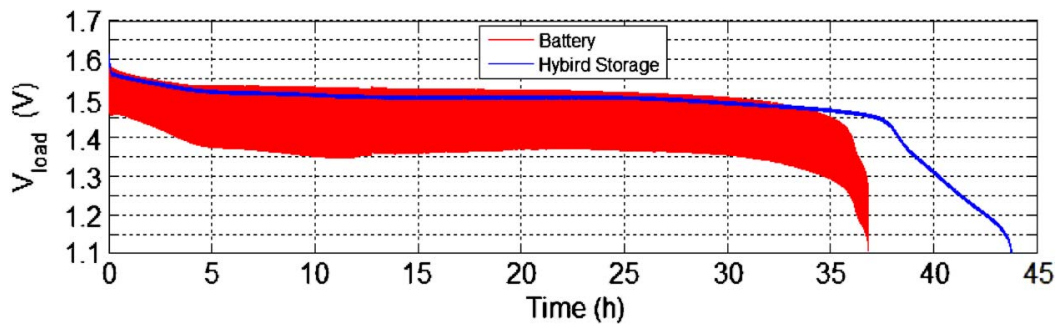

Fig. 10. Discharge profile with the silver oxide battery and the corresponding hybrid-storage unit. The source GS610 was used as load.

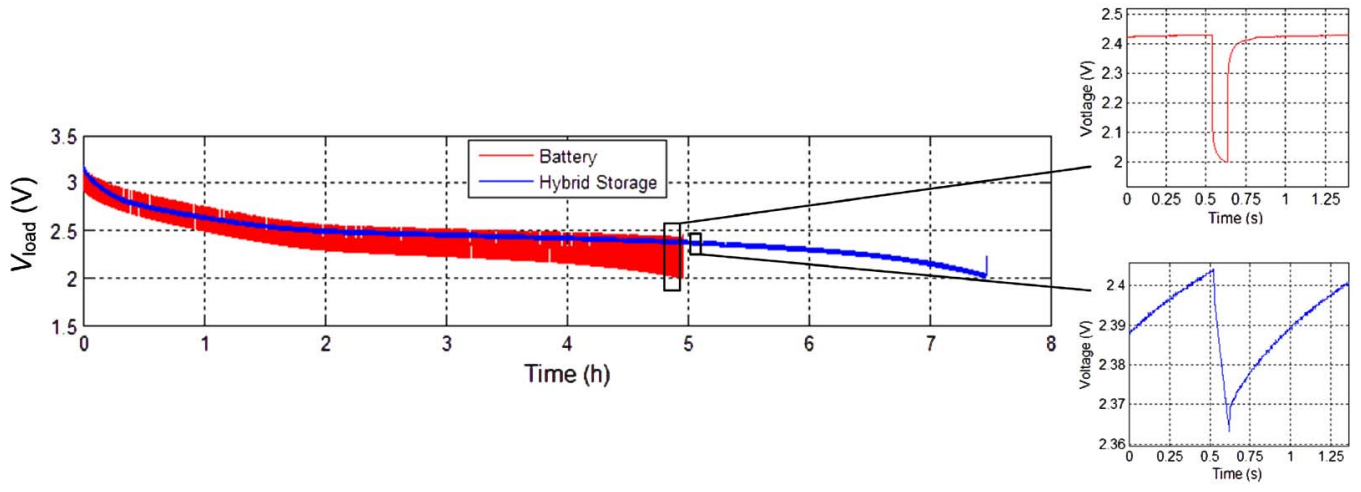

Fig. 11. Discharge profile with the lithium battery and the corresponding hybrid-storage unit. The source GS610 was used as load.

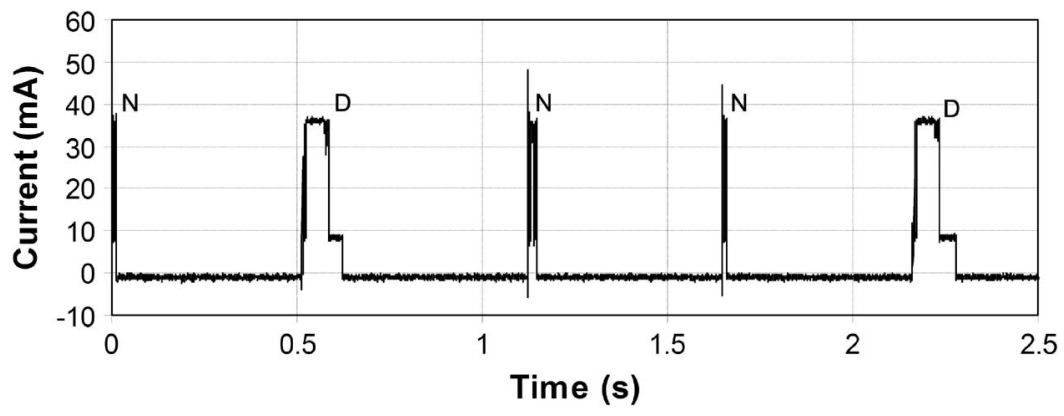

Fig. 12. Current-consumption profile of the sensor node.

GS610 was used as load. As can be seen, the runtime depends on the value of $V_{\mathrm{T}}$. For the single battery, the runtime is very dependent on $V_{\mathrm{T}}$ and ranges from only $1 \mathrm{~h}$ for $V_{\mathrm{T}}=1.45 \mathrm{~V}$ (thus, a lot nonprofitable charge still remains at the battery) to more than $34 \mathrm{~h}$ for $V_{\mathrm{T}}=1.3 \mathrm{~V}$. The large voltage drop that roughly ranges from 150 to $200 \mathrm{mV}$ is due to the internal impedance of the battery. For the hybrid-storage unit, the runtime did not significantly change for $V_{\mathrm{T}}<1.45 \mathrm{~V}$ and was always higher than $37 \mathrm{~h}$. Thus, the addition of a capacitor very much helps in squeezing the battery. The prior sudden decrease in the battery voltage with the single battery (after $35 \mathrm{~h}$ of runtime) can be due to slight differences between the two different primary batteries used for the two different storage units.

Fig. 11 shows the results with the lithium battery and the source GS610. For $V_{\mathrm{T}}=2 \mathrm{~V}$, the runtimes were $4 \mathrm{~h} 58 \mathrm{~min}$ and $7 \mathrm{~h} 28 \mathrm{~min}$ for the single battery and the corresponding hybridstorage unit, respectively. The runtime of the hybrid-storage unit matches the discharge time specified by the manufacturer for a continuous discharge current of $3 \mathrm{~mA}$ (7 h $30 \mathrm{~min})$. As can be inferred from the graph corresponding to the single battery, $R_{\mathrm{B}}$ increased with a decreasing SoC. In the hybridstorage unit, the increasing value of $R_{\mathrm{B}}$ has no effect, because the much lower value of $R_{\mathrm{C}}$ dominated the overall contribution to the voltage drop. The two insets show the voltage profile during the active time for the single battery (upper inset) and the hybrid-storage unit (lower inset). A much larger voltage drop is observed with the single battery $(\sim 0.4 \mathrm{~V})$ than with the hybridstorage unit $(\sim 40 \mathrm{mV})$.

Fig. 12 shows the current-consumption profile of the sensor node (Fig. 9) recorded during $2.5 \mathrm{~s}$ with an oscilloscope (Agilent MSO8104A). Network function packets (marked with $\mathrm{N}$ ) were sent among data packets (marked with $\mathrm{D}$ ). The current consumption was of: $36 \mathrm{~mA}$ during transmissions, $9 \mathrm{~mA}$ when active and in the microampere level while sleeping. The average current consumption was calculated from a 200-s acquisition frame and resulted in $2.23 \mathrm{~mA}$. Fig. 13 shows the discharge profile with the lithium battery and the corresponding hybrid-storage unit when using the sensor node as load. Temperature data were properly sent until the voltage dropped to $1.65 \mathrm{~V}\left(V_{\mathrm{T}}\right)$. At this point, the Zigbee module stopped 


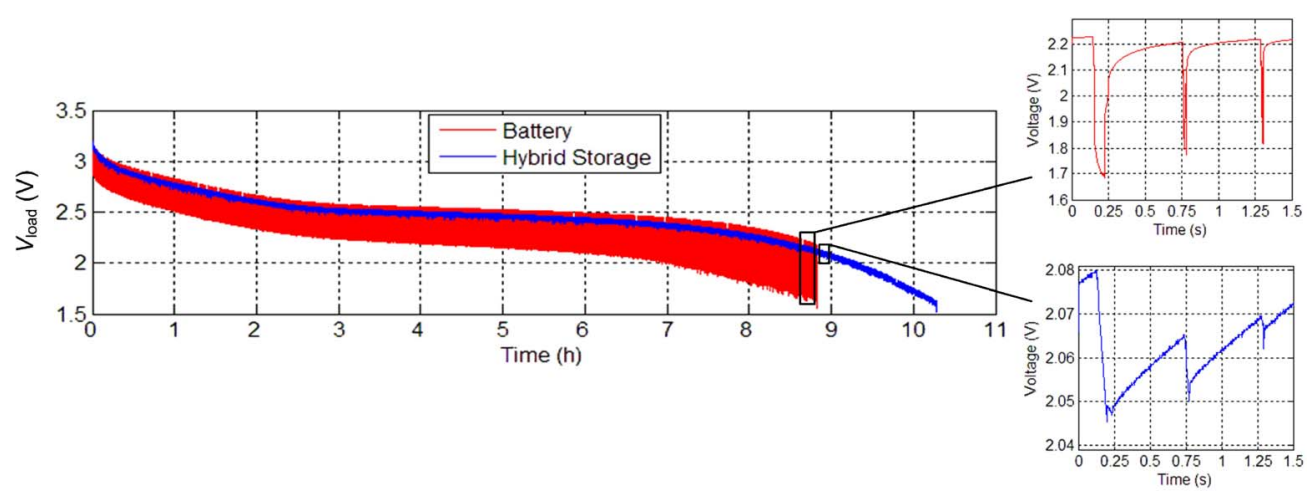

Fig. 13. Discharge profile with the lithium battery and the corresponding hybrid-storage unit. The sensor node was used as load.

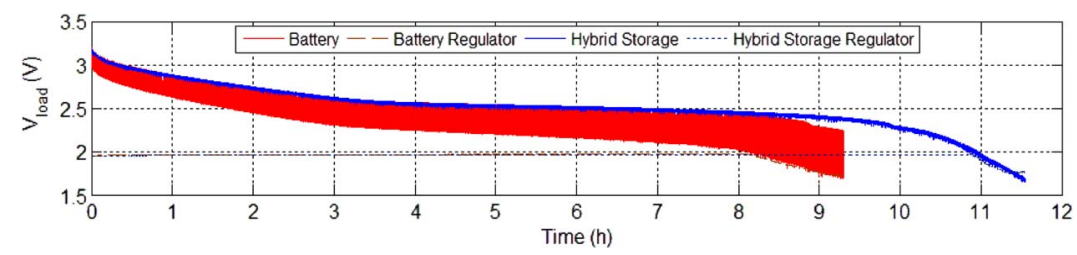

Fig. 14. Discharge profile with the lithium battery and the corresponding hybrid-storage unit. A dc-dc regulator (MAX1920) was added between the storage unit and the sensor node. The voltage at the output of the regulator is also shown.

working properly. This value is quite below the minimum voltage recommended by the manufacturer $(2.1 \mathrm{~V})$. The runtime for the single battery was $8 \mathrm{~h} 50 \mathrm{~min}$. The runtime for the hybridstorage unit increased by $16 \%$, i.e., up to $10 \mathrm{~h} 16 \mathrm{~min}$. This time is in accordance with the discharge curves provided by the manufacturer of the battery for discharge currents between 2 and $3 \mathrm{~mA}$. The amplitude of the voltage drops was similar to that obtained in Fig. 11. On the other hand, the node runtime with both storage units was larger than that in Fig. 11 because of the lower mean current consumption of the load and the lower value resulting for $V_{\mathrm{T}}$. At $-15^{\circ} \mathrm{C}$, the runtime extension achieved was $130 \%$, passing from $2 \mathrm{~h} 44 \mathrm{~min}$ to $6 \mathrm{~h} 18 \mathrm{~min}$ (graphs not shown). Absolute runtimes were reduced with respect to that of Fig. 13 probably due to the reduction in chemical activity and the increase in the internal resistance of the battery at low temperatures [4]. The voltage drop with the single battery increased to $0.9 \mathrm{~V}$.

Fig. 14 extends the results presented in Fig. 13 by adding a MAX1920 buck converter that is configured to provide a regulated output voltage near $2 \mathrm{~V}$. The voltages at the output of the converter are also represented. As can be seen, the converter correctly worked down to an input voltage from the storage unit of $2 \mathrm{~V}$. The runtime up to this point for the single battery increased with respect to that of Fig. 13, passing from $7 \mathrm{~h}$ to $8 \mathrm{~h} 8 \mathrm{~min}$, which is due to the use of the buck converter. The runtime was $10 \mathrm{~h} 51 \mathrm{~min}$ with the hybrid-storage unit, which is almost $2 \mathrm{~h}$ more than that observed in Fig. 13 for $V_{\mathrm{T}}=2 \mathrm{~V}$. Thus, the runtime extension of the hybrid-storage unit with respect to the single battery was now $2 \mathrm{~h} 43 \mathrm{~min}$, which is a $33 \%$ increase and is then higher than that without using a dc-dc converter, as stated in Section IV-C. Clever use of the shutdown functionality of the dc-dc converter could reduce the power consumption during the sleep time intervals of the sensor node. This can be feasible if, for example, the transceiver is powered through the converter and if the microcontroller is directly powered from the battery, providing the shutdown control signal.

\section{CONCLUSION}

Batteries, which are either primary or secondary, are used to power the sensor nodes of WSNs. These nodes remain inactive most of the time to save power. Whenever the load wakes up, a significant voltage drop can appear across the battery terminals, thus preventing the extraction of all the available energy from the battery and leading to a reduction in the node runtime. Additionally, energy is lost at the internal impedance of the battery.

A hybrid-storage unit formed by the parallel combination of a battery and a supercapacitor has been used to extend the runtime of low-power wireless sensor nodes. A comprehensive theoretical analysis that extends previous works found in the literature has been presented. Sleep and leakage currents have been considered, and design guidelines for choosing the appropriate capacitor have been provided. From the analysis, it has been shown that supercapacitors with a low serial resistance mainly help when using high-resistance batteries; thus, they allow the use of batteries that, a priori, would be ruled out.

Three low-capacity batteries $(<200 \mathrm{mAh})$ have been selected: 1) a primary silver oxide battery; 2 ) a secondary lithium; and 3) a secondary NiMH. For the NiMH battery, the addition of a supercapacitor did not provide any improvement, because its internal impedance was already low enough. For the remaining two batteries, suitable capacitors have been selected. Tests when using an electronic load as a pulsed current sink have been performed. The hybrid-storage units always achieved a higher runtime than their single-battery counterparts. The performance of a wireless sensor node has also been tested with the lithium 
battery. The addition of a 0.1-F supercapacitor increased the runtime by $16 \%$. When adding a dc-dc regulator, the increase was $33 \%$. The supercapacitor leakage was lower than $3 \mu \mathrm{A}$ at RT, which is on the same order of magnitude as the sleep current of commercial transceivers. Because the active currents of transceivers are about $30 \mathrm{~mA}$, only for $D<10^{-4}$ will the leakage currents be dominant.

\section{ACKNOWLEDGMENT}

The authors would like to thank J. Albesa for the technical support in the setup of the wireless sensor node and F. López for the technical support in the implementation of the printed circuit boards.

\section{REFERENCES}

[1] F. Rahman, A. Kumar, G. Nagendra, and G. S. Gupta, "Network approach for physiological parameters measurement," IEEE Trans. Instrum. Meas., vol. 54, no. 1, pp. 337-346, Feb. 2005.

[2] Y. Kim, R. G. Evans, and W. M. Iversen, "Remote sensing and control of an irrigation system using a distributed wireless sensor network," IEEE Trans. Instrum. Meas., vol. 57, no. 7, pp. 1379-1387, Jul. 2008.

[3] M. T. Penella and M. Gasulla, "A review of commercial energy harvesters for autonomous sensors," in Proc. IMTC, Warsaw, Poland, May 1-3, 2007, pp. $1-5$

[4] D. Linden, Handbook of Batteries, 2nd ed. New York: McGraw-Hill, 1995.

[5] G. A. Rincon-Mora and M. Chen, Squeezing Operational Life Out of a Shrinking Energy Capsule. London, U.K.: Power Management Design Line, United Business Media LLC, Nov. 20, 2006. [Online]. Available: http://www.powermanagementdesignline.com/ howto/194500129

[6] T. Christen and M. W. Carlen, "Theory of Ragone plots," J. Power Sources, vol. 91, no. 2, pp. 210-216, Dec. 2000.

[7] Tadiran Batteries, PulsesPlus Batteries for High Current Pulse Applications, Sep. 1, 2009. [Online]. Available: http://www.tadiran.com/ index.php/pulses-plus-lithium-cells

[8] T. A. Smith, J. P. Mars, and G. A. Turner, "Using supercapacitors to improve battery performance," in Proc. PESC, 2002, pp. 124-128.

[9] R. A. Dougal, S. Liu, and R. E. White, "Power and life extension of battery-ultracapacitor hybrids," IEEE Trans. Compon. Packag. Technol., vol. 25, no. 1, pp. 120-131, Mar. 2002.
[10] C. E. Holland, J. W. Weidner, R. A. Dougal, and R. E. White, "Experimental characterization of hybrid power systems under pulse current loads," J. Power Sources, vol. 109, no. 1, pp. 32-37, Jun. 2002.

[11] M. T. Penella and M. Gasulla, "Battery squeezing under low-power pulsed loads," in Proc. I2MTC, Victoria, BC, Canada, May 12-15, 2008, pp. 1184-1188.

[12] J. Schindall, "The charge of the ultracapacitors," IEEE Spectr, vol. 44, no. 11, pp. 42-46, Nov. 2007.

[13] Telegesis, Products. Telegesis ZigBee Technology, Aug. 2007. [Online]. Available: http://www.telegesis.com/Zigbee/zproducts.htm

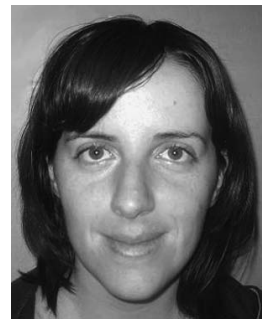

Maria T. Penella (S'07) received the B.Sc. and M.Sc. degrees in telecommunication in 2003 and 2005, respectively, from the Universitat Politècnica de Catalunya (UPC), Barcelona, Spain, where she is currently working toward the Ph.D. degree.

She is currently an Assistant Professor with the Instrumentation, Sensors and Interfaces Group, UPC. In 2007, she was with the Laboratory of Electronics, École Polytechnique Fédérale de Lausanne, Lausanne, Switzerland. Her research interests include energy harvesting, energy and power conditioning, storage elements, and wireless sensor networks.

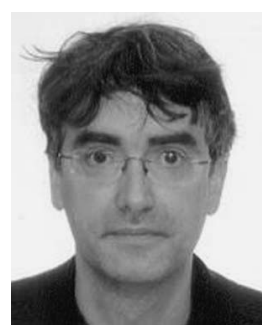

Manel Gasulla (M'01) received the Ingeniero (M.Eng.) and Doctor Ingeniero (Ph.D.) degrees in telecommunication from the Universitat Politècnica de Catalunya (UPC), Barcelona, Spain, in 1992 and 1999, respectively.

Since 1993, he has been with the Instrumentation, Sensors and Interfaces Group, UPC, where he is currently an Associate Professor engaged in teaching analog electronics and electronic instrumentation. During 2001-2002, he was a Visiting Postdoc with the Electronic Instrumentation Laboratory, Delft University of Technology, Delft, The Netherlands. His research interests include capacitive sensors, sensor interfaces and energy harvesting for lowpower autonomous sensors, and wireless sensor networks. 